\title{
Waldbrände im Kreuzfeuer
}

\author{
Marco Moretti* und Marco Conedera
}

\begin{abstract}
Forest Fire in the Cross Fire
Abstract: Since prehistoric times, natural and man made fires were important for forest ecosystem dynamics. This study shows that species richness on the southern slopes of the Swiss Alps was significantly higher on sites with repeated fires than on those, which did not burn. Fast spreading surface fires of low to medium intensity are likely to be crucial for initiating succession and adaptation processes in plants and animals, thus enhancing biodiversity and promoting species conservation. On the other hand, fires pose significant threats to people and goods - through direct damage, erosion or emissions. Therefore, in order to reduce biodiversity loss, it is suggested to introduce silvicultural methods, which mimic sporadic fires through providing a mosaic forest with open gaps of different successional stages.
\end{abstract}

Keywords: biodiversity, chestnut forests, fire ecology, fire history, forest management, invertebrates

Waldbrände zwischen Natur und Kultur

euer ist eines der ältesten Werkzeuge

des Menschen. Der Mensch hat das natürliche Feuergeschehen seit seinem Erscheinen auf der Erde so stark verändert, daß die Feuergeschichte als Produkt der Koevolution von Feuer und Mensch angesehen werden kann ${ }^{[1]}$. Natürlich braucht es dazu auch die Natur: tote oder lebende Biomasse (Brandgut) muß produziert werden, und das Wetter muß günstig sein, damit Feuer entstehen und sich ausbreiten kann (Dürreperioden, Wind).

Bei natürlichen Bränden wird die Zündenergie in Form von Blitzschlag oder Vulkaneruptionen von der Natur geliefert ${ }^{[2]}$. Die meisten Brände in der Welt werden aber vom Menschen entfacht. Fast überall auf der Welt, wo es Brandgut gab, hat der Mensch mit Hilfe des Feuers Wälder gerodet und eine regelrechte Feuerkultur entwickelt. In Afrika und Australien nutzten die Vorfahren der heutigen Bewohner Feuer bereits vor mehreren Jahrtausenden. Zuerst schufen sie freie Flächen für die Jagd und später betrieben sie Wanderackerbau. Diese Art, das Feuer zu nutzen, ist bis heute in vielen Ländern des Tropengürtels in Afrika, Asien und Südamerika verbreitet ${ }^{[3]}$.

In Europa hingegen verlor das Feuer rasch seine Bedeutung für die Landschaftsentwicklung: der schnelle Übergang zu einer seßhaften Landwirtschaft sowie religiöse und kulturelle Werte und Auffassungen veranlaßten die Menschen, die Enstehung größerer Brände zu verhindern. Damit ging die natürliche,

* Postadresse: Dr. M. Moretti

WSL Sottostazione Sud delle Alpi

via Belsoggiorno 22

CH-6504 Bellinzona-Ravecchia (Schweiz)

E-mail: marco.moretti@wsl.ch grundsätzlich positive Beziehung zum Feuer aus früheren Zeiten verloren: Feuer wurde zum Feind erklärt, der dem Menschen nur schadete und bekämpft werden mußte. Diese Einstellung blieb auch erhalten, als durch die Modernisierung der Landwirtschaft viele Randflächen wieder zu Gebüschwald wurden und in der Folge die Zahl der "unerwünschten" Busch- und Waldbrände allmählich wieder zunahm.

Infolge der Auswanderung vieler Europäer verlief diese Entwicklung in Nordamerika ähnlich, wenn auch zeitlich verzögert. Ende des 19. Jahrhunderts nahm das von den ursprünglichen Bewohnern überlieferte Bewußtsein, daß Feuer eine ökologische Bedeutung habe, bei den Siedlern ab. Es ereigneten sich weiterhin einige verheerende Kronenbrände, denen hunderttausende von Bäumen zum Opfer fielen. Im Jahr 1891 berichteten kalifornische Siedler stolz über Rettungsaktionen, mit denen sie in den vorangegangenen Jahren riesige Mammutbäume im uralten $S e$ quoia-Wald vor Waldbränden bewahrt hatten. Der damalige Chef des Forstdiensts Gifford Pinchot stellte daraufhin die Frage: $»$ Wer hat sie in den anderen drei- bis viertausend Jahren ihres Daseins erhalten? « ${ }^{[4]}$. Mammutbäume sind in der Tat durch die dicke Borke vor Feuer geschützt. Sie brauchen aber Feuer, damit die Zapfen sich öffnen und die Samen zu Boden fallen können. Ohne Feuer verjüngt sich also ein Sequoia-Wald nicht. Das Wissen, daß Feuer nützliche Funktionen für die Waldökosysteme haben und systematisches Feuerlöschen diese Funktionen langfristig untergraben kann, blieb zumindest in einigen Gegenden Nordamerikas latent erhalten. Nach dem schrecklichen Brandjahr von 1970, als in den Bundesstaaten Washington und Kalifornien trotz eines riesigen Feuerwehraufgebots Zehntausende Hektar Wald abbrannten, besannen sich viele
Menschen wieder auf die naturgegebene Rolle des Feuers. Studien über die ökologische Bedeutung des Feuers gewannen an Bedeutung und die Feuerökologie wurde zu einer Wissenschaft. In den siebziger Jahren des 20. Jahrhunderts entstanden in Nordamerika Ideen und Konzepte wie das Feuermanagement - das an die Stelle des systematischen Feuerlöschens trat - und das absichtliche Legen präventiver Brände. In der Folge faßten diese Vorstellungen langsam auch in Europa Fuß.

Ende des 20. Jahrhunderts schließlich wurden Waldbrände als ein globales Problem erkannt. Einerseits übertrugen die Medien Berichte und atemberaubende Bilder bedrohlicher Waldbrände aus Australien, Amerika oder Rußland innerhalb kürzester Zeit in jede Stube; anderseits sind spätestens seit der Konferenz für Umwelt und Entwicklung in Rio de Janeiro 1992 immer mehr Menschen bezüglich Umweltveränderungen hellhörig geworden. Tatsache ist, daß die Folgen von Waldbränden seitdem international auch im Hinblick auf Immissionen, Klimaveränderungen und die Gesundheit von Lebewesen sensibel wahrgenommen und intensiv diskutiert werden ${ }^{[5]}$. Waldbrände gerieten ins Kreuzfeuer. Die Diskussion der Frage, ob Feuer vorwiegend positive ökologische Wirkungen oder negative Folgen für die Gesellschaft habe ist kontrovers. In diesem Beitrag diskutieren wir die Situation in der Schweiz, basierend auf einer bald zehnjährigen Forschungserfahrung.

\section{Feuer ist nicht gleich Feuer}

Daß Feuer nicht gleich Feuer ist, wird daran deutlich, daß sich etwa Waldbrände nach ihrer Typologie klassifizieren lassen: man unterscheidet Bodenfeuer (mottende Feuer, welche das unterirdische Brandgut langsam verbrennen), Lauffeuer (schnell voranschreitende Oberflächenfeuer, die nur das Brandgut auf der Bodenoberfläche verbrennen, Figur 1) und Kronenfeuer (Feuer mit heftigen Flammen, die bis zum Kronenbereich der Bäume reichen). Es gibt jedoch auch Kombinationen dieser Typen ${ }^{[6]}$. Je nach Wetterlage und Brandtypologie können Waldbrände mit ganz unterschiedlicher Intensität, Saisonalität und Häufigkeit auftreten. Die Kombination dieser drei Faktoren nennt man das Feuerregime.

Bei hoher Feuerbelastung (häufige und intensive Brände) überleben normalerweise nur Arten, die sich an Feuer angepaßt haben. Arten, die sich nach einem Feuer nur langsam und mühsam erholen und fortpflanzen, laufen Gefahr, dabei ausgerottet $\mathrm{zu}$ werden. Entscheidend für 
Figur 1. Feuer ist nicht gleich Feuer: Lauffeuer breiten sich oft sprunghaft aus und hinterlassen unverbrannte Inseln, von denen aus mobile Arten die Brandflächen wieder neu kolonisieren. Typisches Lauffeuer während der Wintersaison im Kastaniengürtel der Alpensüdseite. (Photo: WSL, Bellinzona)

die Beurteilung der Folgen von Waldbränden ist somit nicht das einzelne Ereignis, entscheidend sind vielmehr auch die Feuergeschichte und das Feuerregime des Gebietes.

\section{Die Waldbrandsituation in der Schweiz}

Im August 2003 zerstörte ein Waldbrand oberhalb des Walliser Dorfes Leuk etwa 350 Hektar Wald. Mehr als 50 Hektar davon waren Schutzwälder, in denen nun kurzfristig Lawinenverbauungen erstellt werden müssen, um die Kantonsstraße und das Dorf vor Lawinen und Rutschungen zu schützen ${ }^{[7]}$. Ereignisse dieser Größenordnung treten in den Wäldern des Schweizer Alpenraums sehr selten auf, so daß Feuer für den Gebirgswald nicht als genereller Risikofaktor gilt. Trotzdem zeigen neuere Studien, daß durch Blitzschlag ausgelöste natürliche Brände nicht nur in der Südschweiz ${ }^{[8]}$, sondern auch in den Alpen bereits vor der Besiedelung durch den Menschen auftraten ${ }^{[9]}$. Im Gegensatz zum Alpenraum (im engeren Sinne) verursachte der Mensch auf der feueranfälligeren Alpensüdseite seit dem Neolithikum (ab etwa 5000 vor Christus) immer wieder Feuer im Wald. Die hohe Feuerfrequenz (Figur 2) geht auf die Brandrodung zurück, die während der Bronze- und Eisenzeit ihre größte Verbreitung erreichte. Die Einführung der Kastanien durch die Römer führte dann zu dauerhaften Kulturen und damit zur

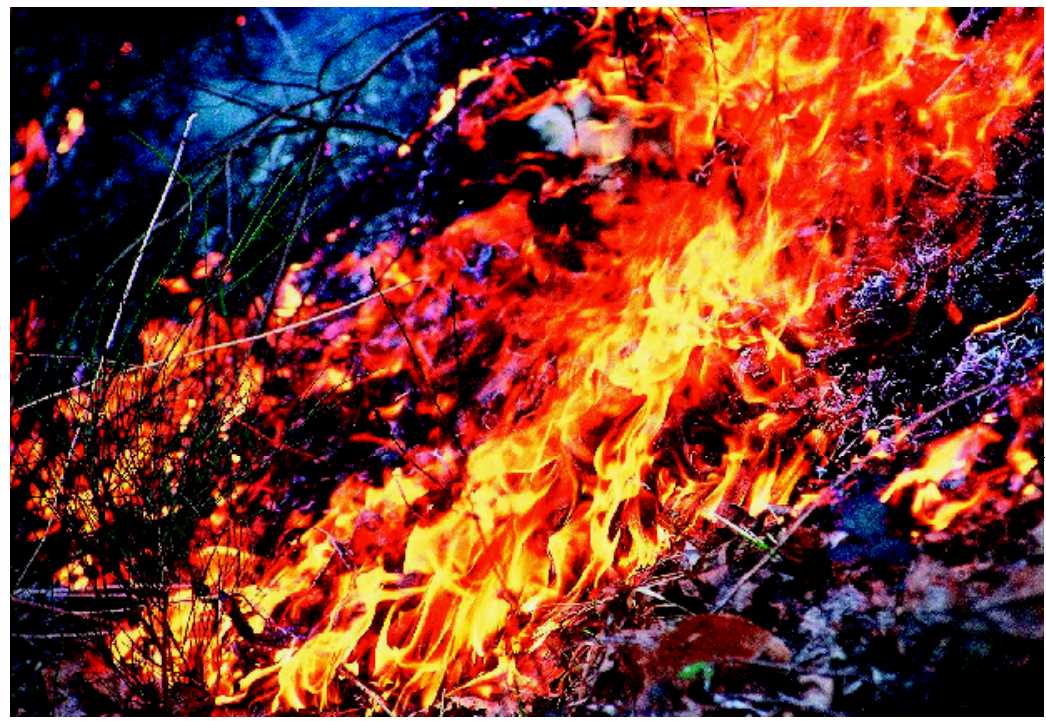

Einstellung der Brandrodung. Dies hatte zur Folge, daß die Feuerfrequenz sank (Figur 2) ${ }^{[8]}$.

Bis Mitte des 20. Jahrhunderts wurde in der Schweiz, ähnlich wie in anderen mitteleuropäischen Ländern, die auf dem Waldboden liegende Biomasse durch aktive Streu- und Holznutzung niedrig gehalten. Seit einigen Jahrzehnten nehmen natürliche und anthropogene Waldbrände infolge der Extensivierung der Forst- und Landwirtschaft generell wieder zu ${ }^{[9]}$. Welche Folgen diese Entwicklung für eine alpine Landschaft haben kann, ist noch weitgehend unbekannt. Seit einigen Jahren arbeiten deshalb Forschungsgruppen aus verschiedenen Institutionen darunter auch die Sottostazione Sud delle Alpi der Eidgenössichen Forschungsanstalt für Wald, Schnee und Landschaft (WSL) - an dieser Thematik. Der Kastaniengürtel auf der Alpensüdseite ist dafür eine ideale Forschungsregion, denn die sich nur wenige Zentimeter darunter das

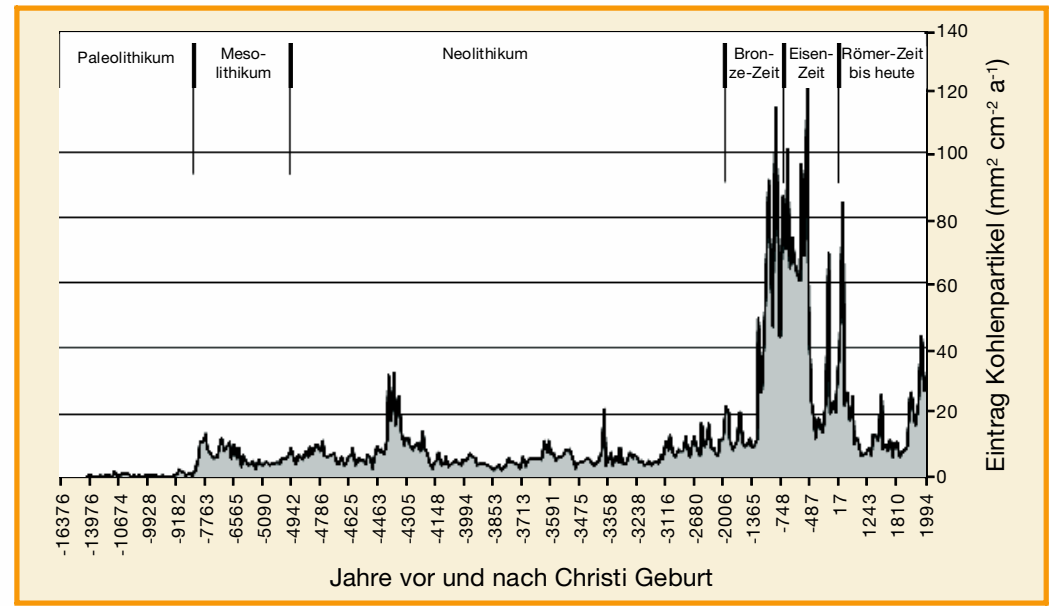

klimatischen Bedingungen und die frühe Aufgabe der Kastanienkultur ließen die Waldbrandfrequenz seit den Sechziger Jahren des 20. Jahrhunderts besonders ansteigen ${ }^{[10]}$. Im Gegensatz zum Mittelmeerraum ereignen sich außerdem die meisten Waldbrände an den Südhängen des Alpenbogens in den trockenen Wintermonaten, insbesondere zwischen Januar und April. Es handelt sich dabei vorwiegend um Oberflächenfeuer (Lauffeuer), bei denen die Feuerfront schnell voranschreitet. Dadurch entstehen oberirdisch und an der Bodenoberfläche kurzfristig sehr hohe Temperaturen, während Feuer kaum noch auswirkt. Lauffeuer breiten sich zum Teil sprunghaft aus, weshalb auf größeren Brandflächen immer wieder unverbrannte Inseln verbleiben.

\section{Feuer bedeutet selektive Störung}

Feuer ist in erster Linie eine Störung, die ein Waldökosystem aus dem momentanen Gleichgewicht bringt und Biomasse vernichtet. Diese Störung erfolgt selektiv. Während eines Waldbrandes entwickelt sich in der Regel eine starke Hitze, die als Thermik aufsteigt. Die hohen Temperaturen wirken sich schädlich auf lebendes Gewebe aus. Bei den Pflanzen verbrennen die empfindlichen, unverholzten Teile meist völlig, und das

Figur 2. Langfristige Feuergeschichte rekonstruiert aufgrund des KohlepartikelEintrags in die Seesedimente des Lago di Origlio im südlichen Tessin ${ }^{[8]}$. 
Wachstumsgewebe (Kambium) der glattrindigen Stammteile verholzter Strauchund Baumarten erleidet schwere Schäden. Nur dickborkige Baumarten wie Eichen, alte Birken und Kastanien schaffen es dank der isolierenden Wirkung der Borke - oberirdisch unbeschädigt zu überleben. Die unterirdischen Pflanzenteile hingegen werden kaum vom Oberflächenfeuer beeinträchtigt. Arten wie die Kastanie, der Adlerfarn und das Pfeifengras, die sich stark vegetativ vermehren und aus ihren Wurzelorganen schnell wieder austreiben können, sowie in der Samenbank überdauernde und vom Wind verbreitete Arten sind damit klar im Vorteil. Aus den Samen, die das Feuer im Schutz des Bodens überlebt haben, keimen rasch neue Pflanzen. Diese haben gegenüber Arten, die von außerhalb des verbrannten $\mathrm{Ge}$ bietes kommen, einen Wachstumsvorsprung.

Die selektive Wirkung des Feuers verstärkt sich bei einer höheren Feuerfrequenz: durch die wiederholten Brände verringert sich meistens der Deckungsgrad der Baumschicht und das Innenklima der Vegetation wird unausgeglichener. Zudem fließt ein Teil der Asche mit den Niederschlägen $\mathrm{ab}$, wodurch dem Boden Nährstoffe entzogen werden. Bei hohen Feuerfrequenzen dominieren zunächst wenige, eher anspruchslose Pflanzenarten, die mittels unterirdischer Organe überleben und die schnell neue Triebe bilden. Solche Pflanzen haben oft Pioniercharakter. Im Bereich der Kastanienwälder gehören vor allem die Edelkastanie, die Hänge-Birke, die Robinie, der Adlerfarn, das Pfeifengras und der Besenginster zu den schnellen Wiederbesiedlern südalpiner Waldbrandflächen ${ }^{[11]}$.

Je nach Lebens- und Verhaltensweise reduziert das Feuer auch die Individuenzahl vieler wirbelloser Arten: Am meisten beeinträchtigt sind die Arten, die in der Streuschicht überwintern oder leben (z. B. Spinnen, Ameisen, Wanzen und verschiedene Käferfamilien). In einem von der WSL 1998 ausgeführten Feuerexperiment nahm die Population dieser Wirbellosen durch das Feuer um bis zu 90 Prozent ab. Hingegen kamen Arten, die hauptsächlich in den tiefsten und besser isolierten Streuschichten überwintern (z. B. Asseln, Tausendfüßler) sowie fliegende Insekten (Bienen und Wespen, Bock- und Prachtkäfer usw.) kaum zu Schaden ${ }^{[12]}$. Lauffeuer verbrennen ein Gebiet jedoch in der Regel sehr heterogen und vermögen nur selten eine Art auszulöschen. Dank der Mobilität vieler Arten wird die Brandfläche von den Inseln ausgehend wieder kolonisiert.

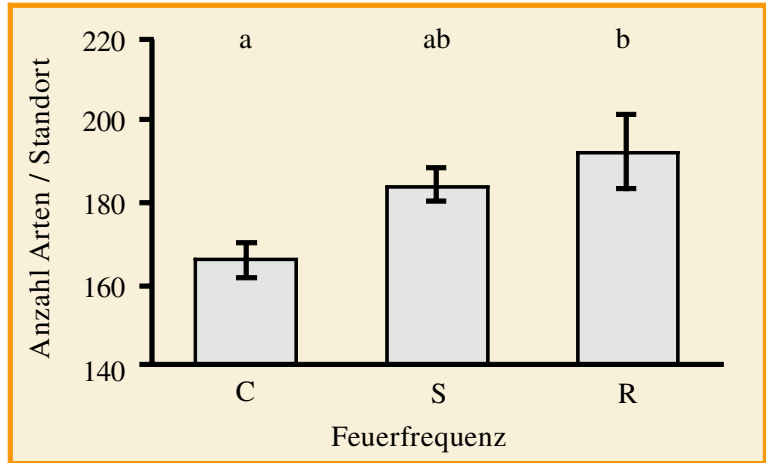

Figur 3. Gesamtzahl der wirbellosen Arten (1085 Arten) in Kastanienniederwäldern der der Schweiz, die in den letzten 30 Jahren nie (C), einmal (S) oder mehr als dreimal (R) gebrannt haben ${ }^{[34]}$. Verschiedene Buchstaben bedeuten statistisch signifikante Unterschiede der Resultate (ANOVA mit Scheffé post-hoc-Test).

\section{Biodiversität nach Feuer}

Feuer reduziert im Ökosystem Wald einerseits die Individuenzahl und die Überlebenschancen feuerempfindlicher Arten. Andererseits schafft es aber neue Lebensbedingungen, indem es zum Beispiel die Biomasse reduziert und eine lichtere Waldstruktur schafft. In der Summe hängen diese Wirkungen davon ab, wie stark das Feuer die Lebensbedingungen (Habitat und Ressourcen) verschiedener Tier- und Pflanzenarten verändert ${ }^{[13]}$. Bei den wirbellosen Tieren beispielsweise führen einzelne Feuerereignisse nicht zu einer signifikanten Erhöhung der Artenzahl. Ihr Lebensraum erholt sich innerhalb kurzer Zeit und bleibt so im wesentlichen unverändert. Hingegen bewirken wiederholte Brände ( $a b$ einer Feuerfrequenz von drei Bränden innerhalb von 30 Jahren) eine bedeutende Zunahme der Artenvielfalt (Figur 3). Bei wiederholten Bränden ändern sich die Waldstrukturen wesentlich, sie werden heterogener und können somit eine größere Vielfalt an Tierarten beherbergen. Wälder, die oft durch Feuer heimgesucht werden, brauchen länger, bis sie wieder das homogenere, strukturund artenärmere Altersstadium erreichen. Eine hohe faunistische Biodiversität bleibt dort also länger erhalten als in Wäldern, die selten brennen (Figur 4).
Das entspricht der intermediate disturbance hypothesis, nach der sich die Anzahl der Arten durch eine Störung mittlerer Intensität und Häufigkeit erhöht ${ }^{[14]}$. Qualitativ gesehen profitieren vor allem Waldrandarten und gefährdete Arten von neuen, vom Feuer geschaffenen Habitaten. Die Anzahl charakteristischer Waldarten hingegen bleibt eher unverändert [15]. Waldrandarten und Arten offener Wälder sind übrigens auch in Wäldern, in denen es seit mehr als 30 Jahren nicht mehr brannte, reichlich vorhanden (Figur 5). Dies erlaubt die Hypothese, daß die Fauna der Kastanienwälder der Alpensüdseite stark an externe Störungsereignisse angepaßt ist.

Ganz anders sind die Wirkungen auf die Vegetation, sei es, weil sie immobil ist, sei es, weil vor allem die Baumarten längere Lebenszyklen und langsamere Reaktionsmöglichkeiten haben. Dennoch nimmt nach einem Feuer die Artenzahl in der Regel zu, vor allem die der lichtbedürftigen Arten. Dieser Effekt ist aber kurzfristig. Im Falle von wiederholten Bränden geht die Artenzahl meistens, ähnlich wie nach Windwurf oder Kahlschlag, nach einer ersten kurzfristigen Zunahme wieder auf ein stabiles Niveau zurück. Dies betrifft vor allem die feuerempfindlichen Baumarten ${ }^{[16]}$.

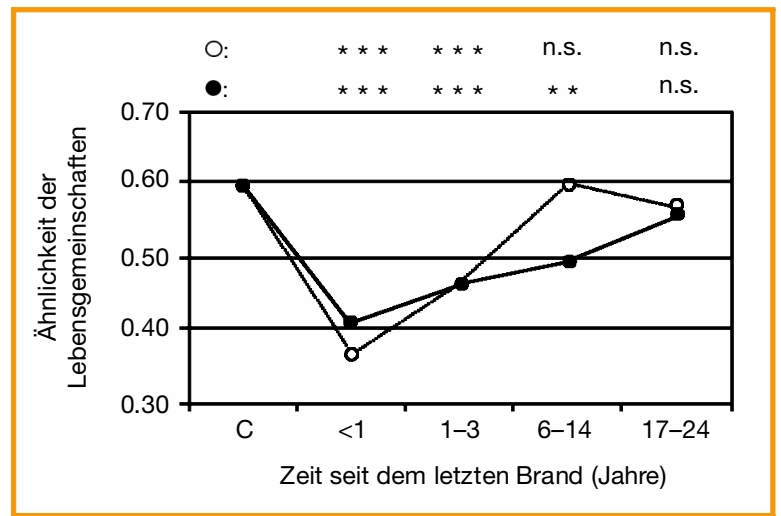

Figur 4. Verlauf des Ähnlichkeitsindexes (nach van der Maarel) der Wirbellosenfauna nach einem $(O)$ oder mehreren Bränden (๑) in Kastanienniederwäldern der Alpensüdseite der Schweiz ${ }^{[35]}$. C = Ähnlichkeit von Flächen ohne Brandereignis, Punkte mit Zeitangaben = Ähnlichkeit von Flächen verschiedenen Brandalters mit brandlosen Flächen. Die Signifikanzen beziehen sich auf die Unterschiede zwischen Brandflächen und den brandlosen Kontrollflächen. ANOVA (O) F4, $42=176.85$, $\mathrm{P}<0.001 ;(\bullet) \mathrm{F} 4,42=106.10$, $\mathrm{P}<0.001$ 


\section{Feuerliebende Arten in der Schweiz}

Im Rahmen der Forschungsaktivitäten auf der Alpensüdseite wurden mehrere pyrophile Tierarten, also solche, die auf Feuer im Wald angewiesen sind, erstmals in der Schweiz festgestellt. Darunter ist zum Beispiel die Wanzenart Aradus lugubris (Figur 6), die dank spezieller Rauchsensoren Waldbrände aus der Ferne lokalisieren kann und dorthin fliegt, um sich von Pilzarten zu ernähren, die auf Brandflächen besonders häufig sind ${ }^{[17]}$. Das gleiche gilt für die Laufkäferart Sericoda quadripunctatum, eine räuberische Art, die auf Brandflächen leicht Beute macht. Leider erlauben es die lückenhaften allgemeinen Kenntnisse über die Pyrophilen Südeuropas im Moment noch nicht, mehr über die Rolle des Feuers bei dieser hochspezialisierten Art auszusagen ${ }^{[18]}$.

Auch unter den Pflanzen gibt es eine phyrophile Art, deren Hauptverbreitungsgebiet vorwiegend der Mittelmeerraum ist: die Zistrose (Cistus salviifolius) (Figur 6). Sie ist insofern vom Feuer abhängig, als die Keimfähigkeit ihrer Samen durch einen Hitzeschock um ein Mehrfaches zunimmt. Ein Waldbrand begünstigt die Zistrose aber auch indirekt, indem er die konkurrierende Vegetation ausschaltet. Im Tessin findet sich diese Art vor allem auf südexponierten Felspartien im Locarnese. Ihr Verbreitungsgebiet verändert sich in Abhängigkeit vom Feuergeschehen ${ }^{[19]}$.

\section{Verheerende Folgen für verbrannte Hänge}

In Kastanienwäldern verbrennen Oberflächenfeuer die Streuschicht und zum Teil auch die Humusschicht. Als Folge davon werden die Steine an der Oberfläche destabilisiert. Häufig kommen bereits während eines Brandes Steine ins Rollen, was Steinschlag auslösen kann. Die schlimmeren Folgen eines Brandes treten aber erst nach dem Feuer ein. Die fehlen- de Bedeckung durch Boden- und Baumvegetation läßt die Regentropfen direkt auf den Mineralboden prallen. Dadurch wird die Bodenstruktur zerstört. Erosion und Auswaschung sind die Folge. Der nackte Boden trocknet schneller aus, die Bodenoberfläche wird wasserundurchlässig, der Regen neigt dazu, oberflächlich abzufließen und den Boden rillenartig zu erodieren ${ }^{[20]}$. Diese negativen Wirkungen treten vor allem in den ersten Monaten nach einem Feuer und in $\mathrm{Ge}$ bieten auf, die seit längerer Zeit von Waldbränden oder anderen Störereignissen verschont blieben. Das Fehlen von störungsangepaßten, schnell reagierenden Pflanzenarten führt dort dazu, daß der Boden längere Zeit der Erosionsgefahr ausgesetzt ist. In extremen Fällen kann es auch zu Katastrophen wie 1997 in Ronco sur Ascona kommen: Einige Monate nach dem Waldbrand wurde durch einen Starkregen ein Murgang, der im Durchschnitt nur alle 100 Jahre erwartet werden kann, ausgelöst. Dieser verfrachtete große Geschiebemengen ${ }^{[21]}$.

\section{Do not disturb?}

In der Natur existieren kaum statische Gleichgewichte; dynamische Gleichgewichte sind die Regel. Feuer trägt zusammen mit anderen Störfaktoren dazu bei, diese Dynamik und die damit verbundene Artenvielfalt wie auch die ökologischen Wechselbeziehungen zu fördern (siehe dazu auch die Theorien der intermediate disturbance hypothesis ${ }^{[16]}$ und der dynamic equilibrium hypothesis $\left.{ }^{[22]}\right)$. So entstand im Laufe der Jahrtausende die Vielfalt der Arten. Diese garantiert im Sinne der Risikoverteilung langfristig auch die Funktionalität der Ökosysteme ${ }^{[23,24]}$. Die europäischen (und somit auch die schweizerischen) Waldökosysteme stellen in dieser Beziehung keine Ausnahme dar: sie entstanden unter dem Druck zuerst von natürlichen, und
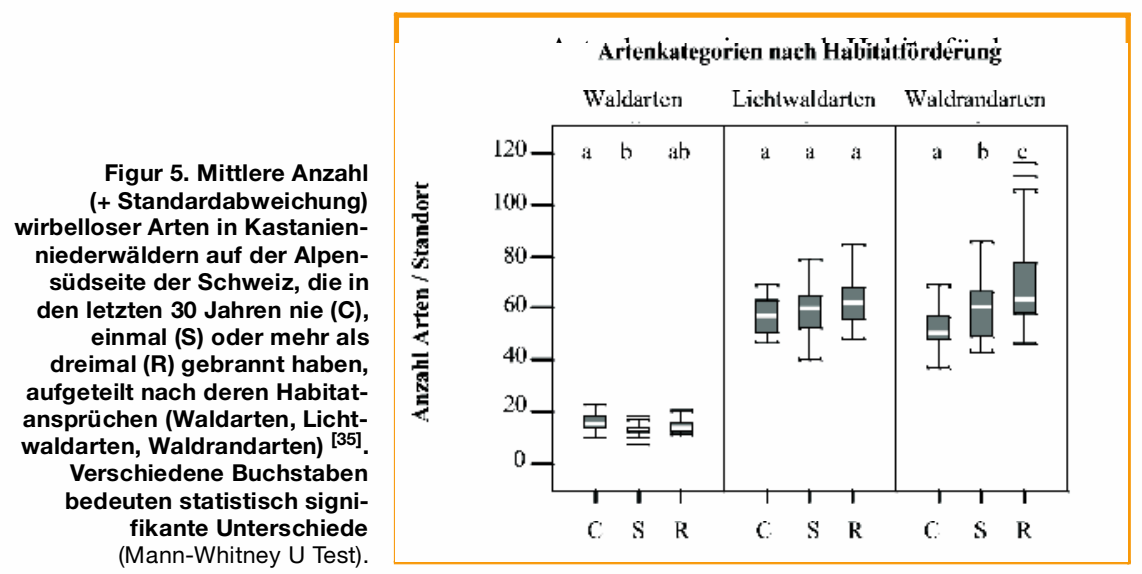

seit dem Neolithikum auch menschlichen Einflußfaktoren ${ }^{[25]}$.

In letzter Zeit wurde die Bedeutung von Naturereignissen wie Windwurf, Feuer, Erdrutschen, aber auch von Holzgewinnung, Flächenräumung und Durchforstung unter dem Gesichtspunkt der "disturbance"-Ökologie, der Biodiversität und des Artenschutzes neu beurteilt ${ }^{\text {[26-30]. }}$ Die entsprechenden Arbeiten betonen den Schutzwert der ersten Sukzessionsphase, in der es häufig große Mengen an Totholz gibt. Das gilt auch für Urwälder, in denen das Stadium der ersten Sukzessionsphase eine andere Artenzusammensetzung aufweist als das des strukturärmeren und generell dunkleren Schlußwaldes ${ }^{[31]}$. Die Dynamik des ständigen, nebeneinander stattfindenden Verjüngens und Alterns fördert viele gefährdete Arten sowohl der frühen als auch der späten Sukzessionsstadien ${ }^{[32]}$.

Die meisten Wälder in feueranfälligen Gebieten am südlichen Alpenhang bestehen aus einem Mosaik von kleinen Brandflächen unterschiedlicher Sukzessionsstadien und großen Flächen von intakten Beständen. In der Tat waren in der Südschweiz in den letzten 30 Jahren nur 10 Prozent der Brandflächen größer als 1.8 Hektar ${ }^{[33]}$. Sowohl für holzbewohnende Arten als auch für solche, die nicht auf Totholz angewiesen sind, scheinen solche Bedingungen ideal zu sein. Viele dieser Arten sind an Störungen angepaßt und profitieren von der Vielfalt verschiedener Umwelt- und Nahrungsbedingungen ${ }^{[17]}$.

Das gleichzeitige Nutzen der positiven Wirkungen von Störungen auf die Walddynamik und die Minimierung der negativen Folgen sind Herausforderungen für Forscher und Fachleute. Es ist naheliegend, daß man in einem alpinen Land wie der Schweiz die zusätzlichen Erosionsund Murganggefahren, die durch Waldbrände entstehen, nicht tolerieren kann. Im relativ dicht erschlossenen und besiedelten Kanton Tessin gefährden Waldbrände auch Menschen und ihre Behausungen. Die Erkenntnisse aus der Feuerökologie dürften trotzdem auch für die Schweiz interessante Hinweise zur Walddynamik liefern, zum Beispiel indem sie Möglichkeiten zur Förderung der Biodiversität aufzeigen. Diese Kenntnisse könnten in die Strategien der Waldbrandbekämpfung oder in künftige waldbauliche Konzepte einfließen. Seltene Arten, die auf ein offenes Habitat angewiesen sind, könnten somit durch aktive waldbauliche Maßnahmen, welche die positiven Effekte des Feuers imitieren, gefördert werden. So sollte in Zukunft angestrebt werden, jene biodiversitäts- 
fördernden Mosaikstrukturen durch waldbauliche Eingriffe zu etablieren, die nach Feuer im Wald entstehen.

\section{Danksagung}

Wir danken Beat Wermelinger und Reinhard Lässig, beide WSL, für ihre Unterstützung bei den Sprachkorrekturen. Den drei anonymen Reviewern sind wir für die interessanten und konstruktiven Bemerkungen dankbar.

\section{Literaturverzeichnis}

[1] S.J. Pyne, P.L. Andrews, D.L. Richard: Introduction to Wildland Fire, John Wiley \& Sons Inc., New York (1996).

[2] W.J. Bond, B.W. van Wilgen: Fire and Plants, Chapman \& Hall, London (1996).

[3] Max-Planck-Gesellschaft: "Spiel mit dem Feuer", GEOMAX 3 (2003) 1-4.

[4] R. Luyken: "Wälder die durchs Feuer gehen", GEO 12 (1989) 64-88.

[5] D. Schwela "Forest fires and their public health impacts: global aspects", III International Conference on Forest Fire Research, Luso, Coimbra, Proceedings, Vol. I (1998) 23-37.

[6] G. Calabri: La prevenzione degli incendi boschivi. I problemi e le tecniche della difesa, Edagricole Bologna, (1991).

[7] Bundesamt für Umwelt, Wald und Landschaft BUWAL: Zerstörter Schutzwald - Bund beteiligt sich an Sofortmassnahmen, Pressemitteilung vom 19. August 2003.

[8] W. Tinner, P. Hubschmid, M. Wehrli, B. Ammann, M. Conedera: "Long-term forest fire ecology and dynamics in southern Switzerland", Journal of Ecology 87 (1999) 273-[29]9.

[9] B. Allgöwer, M. Bur, M. Stähli, N. Koutsias, W. Tinner, M. Conedera, M. Stadler, A. Kaltenbrunner: "Can Long-term Wildland Fire History Help to Design Future Fire and Landscape Management? An Approach from the Swiss Alps", 3rd International Wildland Fire Conference \& Exhibition, Sidney, Proceedings (2003) in press.

[10] M. Conedera: "Incendi di boschi in Canton Ticino: dallo studio pionieristico di Ceschi alla situazione attuale", Bollettino della Società ticinese di Scienze naturali 91 (2003) 135-144

[11] C. Hofmann, M. Conedera, R. Delarze, G. Carraro, P. Giorgetti: "Effets des incendies de forêt sur la végétation au Sud des Alpes suisses", Mitteilungen der Eidgenössischen Forschungsanstalt für Wald, Schnee und Landschaft 73 (1998) 1-90.

[12] S. Herzog: Der direkte Einfluss des Feuers auf die Arthropodenfauna bei einem Waldbrandexperiment im Kanton Tessin, Diplomarbeit ETH Zürich (1998).

[13] M. Moretti, M. Conedera, P. Duelli, P.J. Edwards: "The effects of wildfire on ground-active spiders (Arthropoda: Araneae) in deciduous forests on the southern slope of the Alps", Journal of Applied Ecology 39 (2002) 321-336

[14] J.H. Connell: "Diversity in tropical rain forests and coral reefs, Science 1999 (1978) 1302-1310.

[15] M. Moretti, M.K. Obrist, P. Duelli: "Arthropod biodiversity after forests fires: Winners and losers in the winter fire regime of the Southern Alps", Ecography, submitted.

[16] R. Delarze, D. Calderari, P. Hainard: "Effects of fire on forest dynamics in southern

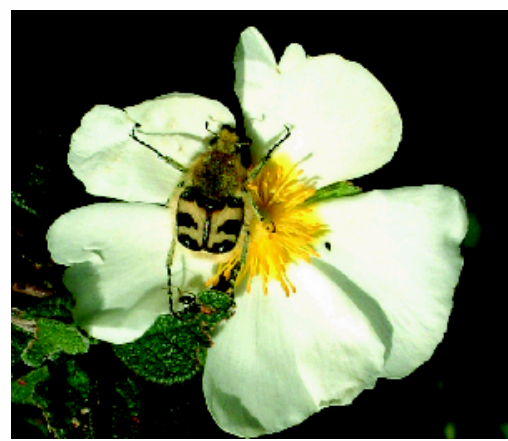

Switzerland", Journal of Vegetation Science 3 (1992) 55-60.

[17] D. Wyniger, M. Moretti, P. Duelli: "Aradus lugubris Fallén, 1807 (Hemiptera, Heteroptera, Aradidae) in a chestnut forest of Southern Switzerland after a fire experiment", Mitteilungen der Schweizerischen Entomologischen Gesellschaft 75 (2002) 173-182.

[18] L-O. Wikars: "Dependence on fire in woodliving insects: an experiment with burned and unburned spruce and birch logs", Journal of Insect Conservation 6 (2002) 1-12.

[19] C. Sini: Le Cistus salviifolius, un arbuste méditerrannéen au Tessin, Diplomarbeit Universität Lausanne (2003).

[20] P. Marxer: "Oberflächenabfluss und Bodenerosion auf Brandflächen des Kastanienwaldgürtel der Südschweiz mit einer Anleitung zur Bewertung der post-fire Erosionsanfälligkeit", Physiogeographica 33 (2003) 1-217.2

[21] M. Conedera, L. Peter, P. Marxer, F. Forster, D Rickenmann, L. Re: "Consequences of forest fires on the hydrogeological response of mountain catchments: a case study of the Riale Buffaga, Ticino, Switzerland", Earth Surface Processes and Landforms 29 (2003) 117-129.

[22] M.A. Hugtson: "A general hypothesis of species diversity", American Naturalist 113 (1979) 81-101.

[23] G. Perterson, C.R. Allen, C.S. Holling: "Ecological resilience, biodiversity, and Scale", Ecosystems 1 (1998) 6-18.

[24] J. Bengtsson: "Disturbance and resilience in soil animal communities", European Journal of Soil Biology 38 (2002) 119-125.

[25] T. Wohlgemuth, M. Bürgi, C. Scheidegger, M. Schütz: "Dominance reduction of species through disturbance - a proposed management principle for central Europe forests", Forest Ecology and Management 166 (2002) 1-15.

[26] B. Wermelinger, P. Duelli, M.K. Obrist, O. Odermatt, M. Seifert: "Die Entwicklung
Figur 6.

Aradus lugubris

(eine Wanzenart, links)

und Cistus salviifolius

(Zistrose, rechts), zwei pyrophile

Arten, die im Tessin vorkommen.

(Photos: Beat Fecker, WSL Birmensdorf;

Giuliano Greco, Gnosca/Tessin)

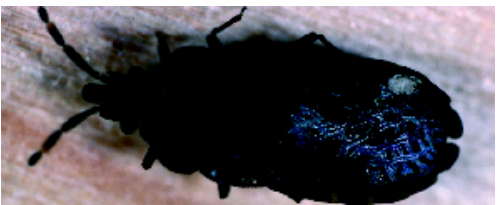

der Fauna auf Windwurfflächen mit und ohne Holzräumung", Schweizerische Zeitschrift für Forstwesen 146 (1995) 913-9 29.

[27] K.J. Kirby, C. Watkins: The Ecological History of European Forests, CAB International, New York (1998)

[28] J. Bengtsson, S.G. Nilsson, A. Franc, P. Menozzi: "Biodiversity, disturbance, ecosystem function and management of European forests", Forest Ecology and Management 132 (2000) 39-50.

[29] A. Floren, K.E. Linsenmair: "The influence of anthropogenic disturbances on the structure of arboreal arthropod communities", Plant Ecology 153 (2001) 153-167.

[30] P.S. White, A. Jentsch: "The search for generality in studies of disturbance and ecosystem dynamics", Progress in Botany 62 (2001) 399-449.

[31] P. Duelli, M.K. Obrist, B. Wermelinger: "Windthrow induces changes of faunistic biodiversity in alpine spruce forests", Forest Snow and Landscape Research 77 (2002) 117-131.

[32] M. Similä, J. Kouki, P. Martikainen, A. Uotila: "Conservation of beetles in boreal pine forests: the effects of forest age and naturalness on species assemblages", Biological Conservation 106 (2002) 19-27.

[33] M. Conedera, M. Marcozzi, B. Jud, D. Mandallaz, F. Chatelain, C. Frank, F. Kienast, P. Ambrosetti, G. Corti: "Incendi boschivi al Sud delle Alpi: passato, presente e possibili sviluppi futuri", Rapporto di lavoro PNR 31, Hochschulverlag AG ETH Zurich (1996).

[34] M. Moretti, M. Zanini, M. Conedera: "Faunistic and floristic post-fire succession in southern Switzerland: an integrated analysis with regard to fire frequency and time since the last fire", Forest Fire Research \& Wildland Fire Safety [CD-ROM] Millpress, Rotterdam (2002).

[35] M. Moretti: Effect of fire on the invertebrate communities in chestnut forests in Southern Switzerland, Dissertation ETH Zürich (2003).

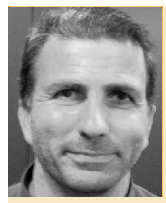

Marco Moretti: Geboren 1961 in Lugano. Studierte Biologie an der Universität Zürich. Danach arbeitete er zehn Jahre lang als selbständiger Ökologe und fertigte parallel seine Dissertation an. Seit August 2002 ist er wissenschaftlicher Mitarbeiter an der Eidgenössichen Forschungsanstalt für Wald, Schnee und Landschaft (WSL) in Bellinzona (Sottostazione Sud delle Alpi).

Marco Conedera: Geboren 1960 in Locarno. Studierte Forstwirtschaft an der ETH Zürich. Nach einer kurzen Zeit freierwerbender Tätigkeit arbeitet er seit 1985 an der WSL. Seit 1991 leitet er die WSL-Außenstelle in Bellinzona (Sottostazione Sud delle Alpi). 